\title{
Multistability in a Multidirectional Associative Memory Neural Network with Delays
}

\author{
Min Wang ${ }^{1}$ and Tiejun Zhou ${ }^{2}$ \\ ${ }^{1}$ College of Orient Science and Technology, Hunan Agricultural University, Changsha, Hunan 410128, China \\ ${ }^{2}$ College of Science, Hunan Agricultural University, Changsha, Hunan 410128, China \\ Correspondence should be addressed to Tiejun Zhou; hntjzhou@126.com
}

Received 29 May 2013; Accepted 2 October 2013

Academic Editor: Qiankun Song

Copyright (c) 2013 M. Wang and T. Zhou. This is an open access article distributed under the Creative Commons Attribution License, which permits unrestricted use, distribution, and reproduction in any medium, provided the original work is properly cited.

This paper focuses on the multidirectional associative memory (MAM) neural networks with $m$ fields which is more advanced to realize associative memory. Based on the Brouwer fixed point theorem and Dini upper right derivative, it is confirmed that the multidirectional associative memory neural network can have $3^{l}$ equilibria and $2^{l}$ equilibria of them are stable, where $l$ is a parameter associated with the number of neurons. Furthermore, an example is given to illustrate the effectiveness of the results.

\section{Introduction}

The Hopfield neural network was introduced by Hopfield in 1982, which introduced the climax of the research on the neural networks. This network was extended to bidirectional associative memory (BAM) neural network by Kosko in 1987 [1] and to multidirectional associative memory (MAM) neural network by Hagiwara in 1990 [2]. They all can realize associative memory. But by using the MAM neural networks, one can achieve the many-to-many association which is a very advanced function of human brain. The many-to-many association has found wide applications in image denoising, speech recognition, pattern recognition, and intelligent information processing [2-6]. For example, it was shown that today most Indians are derived from the two-ancestor group gene by DNA analyzing [7]. If we need to distinguish which category an Indian belongs to, then this is a many-to-many associative problem.

In the designing of the associative memory neural networks to achieve associative memory, it is necessary to ensure their stability. The stability of Hopfield neural networks and BAM neural networks is discussed in a lot of recently published literature works [8-12], but the researchers about MAM neural networks are mainly focused on learning algorithms, fault tolerance, and retrieval efficiency of MAM neural networks [3-6]. To the best of our knowledge, the research on the theory of MAM neural networks was reported only in a few papers [13-18]. Chen et al. proved the stability of some specific types of MAM neural networks in $[13,14]$. We studied the existence and global exponential stability of equilibrium for MAM neural networks with constant delays or time-varying delays in $[15,16]$. We also obtained a sufficient condition for the global exponential stability of the discretetime multidirectional associative memory neural network with variable delays in [17].

The multistability of a neural network describes coexistence of multiple stable patterns such as equilibria or periodic orbits. In recent years, the multistability issue of neural networks is discussed in some papers [18-32]. In [18], we discussed the existence and the exponential stability of multiple periodic solutions for an MAM neural network. The neural networks with a class of nondecreasing piecewise linear activation functions with $2 r$ corner points were investigated in [19]. It was proposed that the $n$-neuron dynamical systems can have and only have $(2 r+1)^{n}$ equilibria under some conditions, of which $(r+1)^{n}$ are locally exponentially stable and others are unstable. A class of neural networks with Mexican-hat-type activation functions was discussed in [20]. A set of new sufficient conditions were presented to ensure the multistability of the neural networks. The cellular neural 
networks with time-varying unbounded delays (DCNNs) were discussed in [21]. Under some conditions, it showed that the DCNNs can exhibit $3^{n}$ equilibrium points. In [22], Huang and Cao found that the $2 n$-dimensional networks can have $3^{n}$ equilibria and $2^{n}$ of them are locally exponentially stable. In [24], with perturbation techniques and the Floquet theory, Campbell et al. discussed the multistability and stable asynchronous periodic oscillations for a network of three identical neurons with multiple discrete signal transmission delays. The possible codimension one bifurcations which occur in the system were determined. In [25], Cheng et al. presented the existence of $2^{n}$ stationary solutions for a general $n$-dimensional delayed neural network with several classes of activation functions. It was shown that a two-dimensional neural network has $n^{2}$ isolated equilibria points which are locally stable, where the activation function has $n$ segments. Furthermore, evoked by periodic external input, $n^{2}$ periodic orbits which are locally exponentially attractive were found [26]. Some similar results were found on $n$-neuron CohenGrossberg neural networks (CGNNs) with time-varying delays and a general class of activation functions [27]. In $[29,30]$, the multistability and multiperiodicity issues were discussed for competitive neural networks and high-order competitive neural networks. In [31], the stability of multiple equilibria of neural networks with time-varying delays and concave-convex characteristics was studied. Some sufficient conditions were obtained to ensure that an $n$-neuron neural network with concave-convex characteristics can have a fixed point located in the appointed region. By partitioning the state space, sufficient conditions were established which ensure that $n$-dimensional Cohen-Grossberg neural networks with $k$-level discontinuous activation functions can have $k^{n}$ equilibrium points or $k^{n}$ periodic orbits [32].

Generally, the existence of a globally stable equilibrium point or periodic solutions is necessary in solving optimization problems, but to achieve many-to-many associative memory by using MAM neural network, the system which has a globally stable equilibrium point or a globally stable periodic solution can only associate less information. So we should study the multistability of MAM neural network in order to make it achieve many-to-many associative memory. It is necessary to explore the existence, stability, and convergence speed of multiple equilibria or periodic solutions of MAM neural network. Motivated by the above, in this paper, we study the multistability issue for a delayed MAM neural network with $m$ fields and $n_{k}$ neurous in the field $k$ as follows:

$$
\begin{aligned}
\frac{d x_{k i}}{d t}= & -a_{k i} x_{k i}(t) \\
& +\sum_{p=1, p \neq k}^{m} \sum_{j=1}^{n_{p}} w_{p j k i} f_{p j}\left(x_{p j}\left(t-\tau_{p j k i}\right)\right)+I_{k i},
\end{aligned}
$$

where $k=1,2, \ldots, m, i=1,2, \ldots, n_{k}, x_{k i}(t)$ denotes the membrane voltage of the $i$ th neuron in the field $k$ at time $t$, $a_{k i}>0$ denotes the decay rate of the $i$ th neuron in the field $k$, $f_{p j}(\cdot)$ is the neuronal activation function of the $j$ th neuron in the field $p, w_{p j k i}$ is the connection weight from the $j$ th neuron in the field $p$ to the $i$ th neuron in the field $k, I_{k i}$ is the external input of the $i$ th neuron in the field $k$, and $\tau_{p j k i}$ is the time delay of the synapse from the $j$ neuron in the field $p$ to the $i$ th neuron in the field $k$.

Set $\tau=\max \left\{\tau_{p j k i} \mid\right.$ where $1 \leq k \leq m, 1 \leq p \leq$ $m, p \neq k, 1 \leq i \leq n_{k}$, and $\left.1 \leq j \leq n_{p}\right\}$. The initial conditions with (1) are of the forms

$$
x_{k i}(t)=\phi_{k i}(t),
$$

where $k=1,2, \ldots, m, i=1,2, \ldots, n_{k}$, and $\phi_{k i}:[-\tau, 0] \rightarrow \mathbb{R}$ are continuous functions.

This paper is organized as follows. In the next section, we discuss the existence of multiple equilibria by using the Brouwer fixed point theorem. In Section 3, we analyze the exponential stability of every equilibrium. An example is given to illustrate the effectiveness of our results in Section 4. Finally, conclusions are given in Section 5.

\section{Existence of Multiple Equilibria}

In this section, we consider the existence of multiple equilibria by using the Brouwer fixed point theorem. Since the existence and stability of stationary patterns for neural networks certainly depend on the characteristics of activation functions, we assume the activation functions $f_{k i}(\cdot)(1 \leq i \leq$ $\left.n_{k}, 1 \leq k \leq m\right)$ satisfy the following condition:

(H1) $f_{k i}(\cdot)$ are continuous, increasing and there exist $M_{k i}>0$ such that $\left|f_{k i}(x)\right| \leq M_{k i}$ for $1 \leq i \leq n_{k}, 1 \leq$ $k \leq m$.

For convenience, we give some notations as follows:

$$
\begin{gathered}
M= \begin{cases}m, & \text { if } m \text { is an even number, } \\
m-1, & \text { if } m \text { is an odd number, }\end{cases} \\
I_{M}=\{1,3,5, \ldots, M-1\}, \\
l_{i}=l_{i+1}=\min \left\{n_{i}, n_{i+1}\right\}, \quad \text { for any } i \in I_{M}, l_{m}=0, \\
\text { if } M \neq m, \\
l=\sum_{i \in I_{M}} l_{i}, \quad n=\sum_{i=1}^{m} n_{i}, \\
\operatorname{col}\left(b_{k i}\right)=\left(b_{11}, \ldots, b_{1 n_{1}}, b_{21}, \ldots, b_{2 n_{2}}, \ldots, b_{m 1}, \ldots, b_{m n_{m}}\right) .
\end{gathered}
$$

Set

$$
\begin{gathered}
\delta_{k i}=\frac{1}{a_{k i}}\left[\sum_{p=1, p \neq k}^{m} \sum_{j=1}^{n_{p}}\left|w_{p j k i}\right| M_{p j}+\left|I_{k i}\right|\right], \\
\Omega=\left\{x=\operatorname{col}\left(x_{k i}\right) \in \mathbb{R}^{n} \mid x_{k i} \in\left[-\delta_{k i}, \delta_{k i}\right],\right. \\
\text { for } \left.1 \leq k \leq m, 1 \leq i \leq n_{k}\right\} .
\end{gathered}
$$




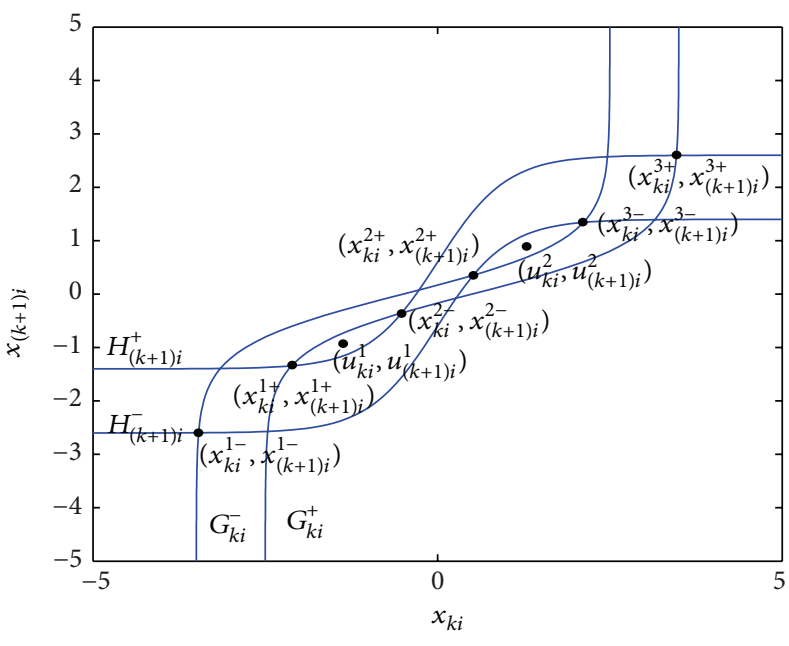

(a)

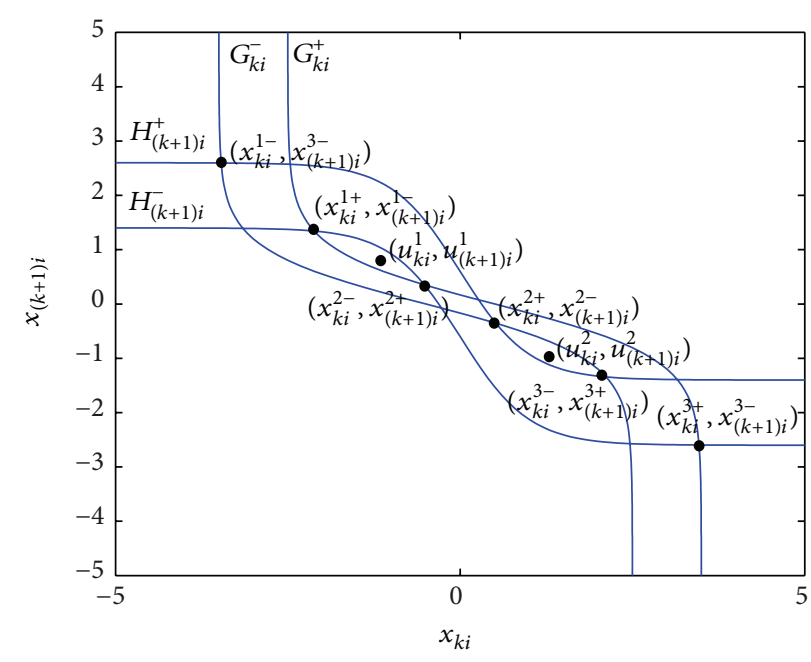

(b)

FIGURE 1: The configurations of $G_{k i}^{+}(y), G_{k i}^{-}(y)$ and $H_{(k+1) i}^{-}(x), H_{(k+1) i}^{+}(x)$. (a) $w_{k i(k+1) i}, w_{(k+1) i k i}>0$. (b) $w_{k i(k+1) i}, w_{(k+1) i k i}<0$.

For any $k \in I_{M}, 1 \leq i \leq l_{k}$, define

$$
\begin{aligned}
& H_{(k+1) i}^{+}\left(x_{k i}\right)=\frac{1}{a_{(k+1) i}}\left(w_{k i(k+1) i} f_{k i}\left(x_{k i}\right)\right. \\
& \left.-\quad\left|w_{k i(k+1) i}\right| M_{k i}\right)+\delta_{(k+1) i}, \\
& H_{(k+1) i}^{-}\left(x_{k i}\right)=\frac{1}{a_{(k+1) i}}\left(w_{k i(k+1) i} f_{k i}\left(x_{k i}\right)\right. \\
& \left.\quad+\left|w_{k i(k+1) i}\right| M_{k i}\right)-\delta_{(k+1) i}, \\
& G_{k i}^{+}\left(x_{(k+1) i}\right)=\frac{1}{a_{k i}}\left(w_{(k+1) i k i} f_{(k+1) i}\left(x_{(k+1) i}\right)\right. \\
& \left.\quad-\left|w_{(k+1) i k i}\right| M_{(k+1) i}\right)+\delta_{k i}, \\
& G_{k i}^{-}\left(x_{(k+1) i}\right)=\frac{1}{a_{k i}}\left(w_{(k+1) i k i} f_{(k+1) i}\left(x_{(k+1) i}\right)\right. \\
& \left.\quad+\left|w_{(k+1) i k i}\right| M_{(k+1) i}\right)-\delta_{k i} .
\end{aligned}
$$

We consider the second parameter condition which is used to establish existence of multiple equilibria for systems (1).

(H2) For any $k \in I_{M}$, the weights $w_{(k+1) i k i} \cdot w_{k i(k+1) i}>$ 0 , and there exist two points $\left(u_{k i}^{1}, u_{(k+1) i}^{1}\right),\left(u_{k i}^{2}, u_{(k+1) i}^{2}\right)$, where $u_{k i}^{1}<u_{k i}^{2}$, such that

(1) if $w_{k i(k+1) i}, w_{(k+1) i k i}>0$, then $H_{(k+1) i}^{+}\left(u_{k i}^{1}\right)<$ $u_{(k+1) i}^{1}, G_{k i}^{+}\left(u_{(k+1) i}^{1}\right)<u_{k i}^{1}, H_{(k+1) i}^{-}\left(u_{k i}^{2}\right)>u_{(k+1) i}^{2}$, $G_{k i}^{-}\left(u_{(k+1) i}^{2}\right)>u_{k i}^{2}$

(2) if $w_{k i(k+1) i}, w_{(k+1) i k i}<0$, then $H_{(k+1) i}^{-}\left(u_{k i}^{1}\right)>$ $u_{(k+1) i}^{1}, G_{k i}^{+}\left(u_{(k+1) i}^{1}\right)<u_{k i}^{1}, H_{(k+1) i}^{+}\left(u_{k i}^{2}\right)<u_{(k+1) i}^{2}$, $G_{k i}^{-}\left(u_{(k+1) i}^{2}\right)>u_{k i}^{2}$.
The configuration that motivates $(\mathrm{H} 2)$ is depicted in Figure 1. Under the above assumptions, if $w_{k i(k+1) i}$, $w_{(k+1) i k i}>0$, then there are three crossing points $\left(x_{k i}^{1+}, x_{(k+1) i}^{1+}\right)$, $\left(x_{k i}^{2-}, x_{(k+1) i}^{2-}\right)$, and $\left(x_{k i}^{3+}, x_{(k+1) i}^{3+}\right)$ of the curves $x_{(k+1) i}=$ $H_{(k+1) i}^{+}\left(x_{k i}\right), x_{k i}=G_{k i}^{+}\left(x_{(k+1) i}\right)$, and there are three crossing points $\left(x_{k i}^{1-}, x_{(k+1) i}^{1-}\right),\left(x_{k i}^{2+}, x_{(k+1) i}^{2+}\right)$, and $\left(x_{k i}^{3-}, x_{(k+1) i}^{3-}\right)$ of the curves $x_{(k+1) i}=H_{(k+1) i}^{-}\left(x_{k i}\right), x_{k i}=G_{k i}^{-}\left(x_{(k+1) i}\right)$ (see Figure 1(a)), where

$$
\begin{aligned}
x_{k i}^{1-}<x_{k i}^{1+} & <u_{k i}^{1}<x_{k i}^{2-}<x_{k i}^{2+}<u_{k i}^{2}<x_{k i}^{3-}<x_{k i}^{3+}, \\
x_{(k+1) i}^{1-} & <x_{(k+1) i}^{1+}<u_{(k+1) i}^{1}<x_{(k+1) i}^{2-} \\
& <x_{(k+1) i}^{2+}<u_{(k+1) i}^{2}<x_{(k+1) i}^{3-}<x_{(k+1) i}^{3+} .
\end{aligned}
$$

If $w_{k i(k+1) i}, w_{(k+1) i k i}<0$, then there are three crossing points $\left(x_{k i}^{1-}, x_{(k+1) i}^{1+}\right),\left(x_{k i}^{2+}, x_{(k+1) i}^{2-}\right)$, and $\left(x_{k i}^{3-}, x_{(k+1) i}^{3+}\right)$ of the curves $x_{(k+1) i}=H_{(k+1) i}^{+}\left(x_{k i}\right), x_{k i}=G_{k i}^{-}\left(x_{(k+1) i}\right)$, and there are three crossing points $\left(x_{k i}^{1+}, x_{(k+1) i}^{1-}\right),\left(x_{k i}^{2-}, x_{(k+1) i}^{2+}\right)$, and $\left(x_{k i}^{3+}, x_{(k+1) i}^{3-}\right)$ of the curves $x_{(k+1) i}=H_{(k+1) i}^{-}\left(x_{k i}\right), x_{k i}=$ $G_{k i}^{+}\left(x_{(k+1) i}\right)$ (see Figure 1(b)), where

$$
\begin{aligned}
x_{k i}^{1-}<x_{k i}^{1+} & <u_{k i}^{1}<x_{k i}^{2-}<x_{k i}^{2+}<u_{k i}^{2}<x_{k i}^{3-}<x_{k i}^{3+}, \\
x_{(k+1) i}^{3-} & <x_{(k+1) i}^{3+}<u_{(k+1) i}^{2}<x_{(k+1) i}^{2-} \\
& <x_{(k+1) i}^{2+}<u_{(k+1) i}^{1}<x_{(k+1) i}^{1-}<x_{(k+1) i}^{1+} .
\end{aligned}
$$


Furthermore, it is easy to confirm that $x_{k i}^{j+}, x_{k i}^{j-} \in$ $\left[-\delta_{k i}, \delta_{k i}\right]$, for any $1 \leq k \leq m, 1 \leq i \leq n_{k}$, and $j=1,2,3$.

Theorem 1. Under conditions (H1) and (H2), there are at least $3^{l}$ equilibria of the multidirectional associative memory neural network (1).

Proof. Set

$$
\begin{array}{r}
\Omega^{\alpha}=\left\{x=\operatorname{col}\left(x_{k i}\right) \in \Omega \mid\left(x_{k i}, x_{(k+1) i}\right)\right. \\
\left.\in \Omega_{k i}^{\alpha_{k i}} \text {, for } k \in I_{M}, 1 \leq i \leq l_{k}\right\},
\end{array}
$$

where $\alpha=\left(\alpha_{11}, \ldots, \alpha_{1 l_{1}}, \alpha_{31}, \ldots, \alpha_{3 l_{3}}, \ldots, \alpha_{(M-1) 1}, \ldots\right.$, $\left.\alpha_{(M-1) l_{M-1}}\right), \alpha_{k i}=1,2$, or 3 , and

$$
\begin{aligned}
\Omega_{k i}^{1}= & \left\{\left(x_{k i}, x_{(k+1) i}\right) \in \mathbb{R}^{2} \mid\left(x_{k i}, x_{(k+1) i}\right) \in\left[x_{k i}^{1-}, x_{k i}^{1+}\right]\right. \\
& \left.\times\left[x_{(k+1) i}^{1-}, x_{(k+1) i}^{1+}\right]\right\}, \\
\Omega_{k i}^{2}= & \left\{\left(x_{k i}, x_{(k+1) i}\right) \in \mathbb{R}^{2} \mid\left(x_{k i}, x_{(k+1) i}\right) \in\left[x_{k i}^{2-}, x_{k i}^{2+}\right]\right. \\
& \left.\times\left[x_{(k+1) i}^{2-}, x_{(k+1) i}^{2+}\right]\right\}, \\
\Omega_{k i}^{3}= & \left\{\left(x_{k i}, x_{(k+1) i}\right) \in \mathbb{R}^{2} \mid\left(x_{k i}, x_{(k+1) i}\right) \in\left[x_{k i}^{3-}, x_{k i}^{3+}\right]\right. \\
& \left.\times\left[x_{(k+1) i}^{3-}, x_{(k+1) i}^{3+}\right]\right\} .
\end{aligned}
$$

Obviously, they are $3^{l}$ disjoint closed regions, and $\Omega_{k i}^{j} \subseteq$ $\left[-\delta_{k i}, \delta_{k i}\right] \times\left[-\delta_{(k+1) i}, \delta_{(k+1) i}\right]$ for any $k \in I_{M}, 1 \leq i \leq l_{k}$, $j=1,2,3$.

For any fixed $\alpha$ and a given $\tilde{x}=\operatorname{col}\left(\tilde{x}_{k i}\right) \in \Omega^{\alpha}$, we study this problem on the following two cases.

Case $1\left(1 \leq i \leq l_{k}\right)$. Consider the following equations:

$$
\begin{aligned}
& -a_{k i} x_{k i}+w_{(k+1) i k i} f_{(k+1) i}\left(x_{(k+1) i}\right)-w_{(k+1) i k i} f_{(k+1) i}\left(\tilde{x}_{(k+1) i}\right) \\
& +\sum_{p=1, p \neq k}^{m} \sum_{j=1}^{n_{p}} w_{p j k i} f_{p j}\left(\tilde{x}_{p j}\right)+I_{k i}=0
\end{aligned}
$$$$
-a_{(k+1) i} x_{(k+1) i}+w_{k i(k+1) i} f_{k i}\left(x_{k i}\right)-w_{k i(k+1) i} f_{k i}\left(\tilde{x}_{k i}\right)
$$$$
+\sum_{p=1, p \neq k}^{m} \sum_{j=1}^{n_{p}} w_{p j(k+1) i} f_{p j}\left(\tilde{x}_{p j}\right)+I_{(k+1) i}=0 .
$$

It follows from (10) that

$$
\begin{aligned}
x_{k i} \leq & \frac{1}{a_{k i}}\left[w_{(k+1) i k i} f_{(k+1) i}\left(x_{(k+1) i}\right)\right. \\
& \left.+\sum_{j=1, j \neq i}^{n_{k+1}}\left|w_{(k+1) j k i}\right| M_{(k+1) j}+\left|I_{k i}\right|\right] \\
+ & \frac{1}{a_{k i}} \sum_{\substack{p=1, p \neq k, j=1 \\
p \neq k+1}}^{m} \sum_{p j i k i}^{n_{p}} \mid M_{p j}
\end{aligned}
$$

$$
\begin{aligned}
& =\frac{1}{a_{k i}}\left[w_{(k+1) i k i} f_{(k+1) i}\left(x_{(k+1) i}\right)\right. \\
& \left.\quad-\left|w_{(k+1) i k i}\right| M_{(k+1) i}+\left|I_{k i}\right|\right] \\
& \quad+\frac{1}{a_{k i}} \sum_{p=1, p \neq k}^{m} \sum_{j=1}^{n_{p}}\left|w_{p j k i}\right| M_{p j} \\
& =\frac{1}{a_{k i}}\left[w_{(k+1) i k i} f_{(k+1) i}\left(x_{(k+1) i}\right)\right. \\
& \left.\quad-\left|w_{(k+1) i k i}\right| M_{(k+1) i}\right]+\delta_{k i} \\
& =G_{k i}^{+}\left(x_{(k+1) i}\right) .
\end{aligned}
$$

Similarly, from (10), we also can obtain that

$$
\begin{aligned}
x_{k i} \geq \frac{1}{a_{k i}} & {\left[w_{(k+1) i k i} f_{(k+1) i}\left(x_{(k+1) i}\right)\right.} \\
& \left.+\left|w_{(k+1) i k i}\right| M_{(k+1) i}\right]-\delta_{k i}=G_{k i}^{-}\left(x_{(k+1) i}\right) .
\end{aligned}
$$

So the curve of (10) is between the curves of $x_{k i}=G_{k i}^{-}\left(x_{(k+1) i}\right)$ and $x_{k i}=G_{k i}^{+}\left(x_{(k+1) i}\right)$.

Under similar analysis we can obtain that the curve of (11) is between the curves of $x_{(k+1) i}=H_{(k+1) i}^{-}\left(x_{k i}\right)$ and $x_{(k+1) i}=$ $H_{(k+1) i}^{+}\left(x_{k i}\right)$. So we can affirm that there are at least three solutions of (10) and (11); each solution lies separately in $\Omega_{k i}^{1}, \Omega_{k i}^{2}$, and $\Omega_{k i}^{3}$. Get one that is in $\Omega_{k i}^{\alpha_{k i}}$, and denote it by $\left(\bar{x}_{k i}, \bar{x}_{(k+1) i}\right)$.

Case $2\left(l_{k}+1 \leq i \leq n_{k}\right)$. Let

$$
\bar{x}_{k i}=\frac{1}{a_{k i}}\left[\sum_{p=1, p \neq k}^{m} \sum_{j=1}^{n_{p}} w_{p j k i} f_{p j}\left(\tilde{x}_{p j}\right)+I_{k i}\right] .
$$

Then

$$
\begin{aligned}
\left|\bar{x}_{k i}\right| & \leq \frac{1}{a_{k i}}\left[\sum_{p=1, p \neq k}^{m} \sum_{j=1}^{n_{p}}\left|w_{p j k i} f_{p j}\left(x_{p j}\right)\right|+I_{k i}\right] \\
& \leq \frac{1}{a_{k i}}\left[\sum_{p=1, p \neq k}^{m} \sum_{j=1}^{n_{p}}\left|w_{p j k i}\right| M_{p j}+\left|I_{k i}\right|\right]=\delta_{k i} .
\end{aligned}
$$

From the two cases above, we can obtain $\bar{x}_{k i} \in \Omega^{\alpha}$ for any given $\tilde{x} \in \Omega^{\alpha}$. Set $\bar{x}=\operatorname{col}\left(\bar{x}_{k i}\right)$. It is obvious that $\bar{x} \in \Omega^{\alpha}$. Set mapping $F^{\alpha}: \Omega^{\alpha} \rightarrow \Omega^{\alpha}$ as follows:

$$
F^{\alpha}(\tilde{x})=\bar{x}
$$

Because $f_{k i}(\cdot)$ are continuous mappings, so $F^{\alpha}$ is continuous mapping. By the Brouwer fixed point theorem, there is at least one fixed point of $F^{\alpha}$, that is, zero point of (1) in $\Omega^{\alpha}$. Therefore, there are at least $3^{l}$ equilibria of the multidirectional associative memory neural network (1). 


\section{Stability Analysis}

In this section, the stability of the equilibria is considered. Set

$$
\begin{aligned}
& \Psi=\left\{\phi=\operatorname{col}\left(\phi_{k i}\right) \in C\left([-\tau, 0], \mathbb{R}^{n}\right) \mid-\delta_{k i} \leq \phi_{k i}(s) \leq \delta_{k i},\right. \\
& \left.\quad \text { for } 1 \leq k \leq m, 1 \leq i \leq n_{k}\right\} \\
& \eta=\left(\eta_{11}, \ldots, \eta_{1 l_{1}}, \eta_{31}, \ldots, \eta_{3 l_{3}}, \ldots, \eta_{(M-1) 1}, \ldots, \eta_{(M-1) l_{M-1}}\right),
\end{aligned}
$$

with $\eta_{k i}=1$ or $\eta_{k i}=3$

We consider the following $2^{l}$ subsets of $\Psi$ :

$$
\begin{gathered}
\Psi^{\eta}=\left\{\phi=\operatorname{col}\left(\phi_{k i}\right) \in \Psi \mid\left(\phi_{k i}, \phi_{(k+1) i}\right) \in \Psi_{k i}^{\eta_{k i}},\right. \\
\text { for } \left.k \in I_{M}, 1 \leq i \leq l_{k}\right\},
\end{gathered}
$$

where

$$
\begin{aligned}
\Psi_{k i}^{1}= & \left\{\left(\phi_{k i}, \phi_{(k+1) i}\right) \in C\left([-\tau, 0], \mathbb{R}^{2}\right) \mid \phi_{p i}(s) \in\left[x_{p i}^{1-}, x_{p i}^{1+}\right],\right. \\
& \text { for } p=k, k+1\}, \\
\Psi_{k i}^{3}=\left\{\left(\phi_{k i}, \phi_{(k+1) i}\right) \in C\left([-\tau, 0], \mathbb{R}^{2}\right) \mid \phi_{p i}(s) \in\left[x_{p i}^{3-}, x_{p i}^{3+}\right],\right. & \text { for } p=k, k+1\} .
\end{aligned}
$$

Lemma 2. Under assumptions (H1) and (H2), each $\Psi^{\eta}$ is a positive invariant set with respect to the solution flow generated by system (1).

Proof. For any given $\eta$ and any initial condition $\phi=\operatorname{col}\left(\phi_{k i}\right) \epsilon$ $\Psi^{\eta}$, let $x(t ; \phi)=\operatorname{col}\left(x_{k i}(t ; \phi)\right)$ be the solution of (1) with initial condition $\phi \in \Psi^{\eta}$.

For any $1 \leq k \leq m, 1 \leq i \leq n_{k}$, and any $t>0$, from (1), we obtain that

$$
-a_{k i} \delta_{k i} \leq \frac{d x_{k i}(t ; \phi)}{d t}+a_{k i} x_{k i}(t ; \phi) \leq a_{k i} \delta_{k i} .
$$

Multiplying both sides of (20) by $e^{a_{k i} t}$, we have

$$
-a_{k i} \delta_{k i} e^{a_{k i} t} \leq \frac{d x_{k i}(t ; \phi) e^{a_{k i} t}}{d t} \leq a_{k i} \delta_{k i} e^{a_{k i} t}
$$

Integrating $(21)$ over $[0, t]$, we obtain

$$
-\delta_{k i}\left(e^{a_{k i} t}-1\right) \leq x_{k i}(t ; \phi) e^{a_{k i} t}-\phi_{k i}(0) \leq \delta_{k i}\left(e^{a_{k i} t}-1\right) .
$$

Hence it follows that, for $t>0$,

$$
\left|x_{k i}(t ; \phi)\right| \leq e^{-a_{k i} t}\left(\left|\phi_{k i}(0)\right|-\delta_{k i}\right)+\delta_{k i} .
$$

Therefore, for any given initial condition $\phi \in \Psi^{\eta}$, we have, for $t>0$,

$$
\left|x_{k i}(t ; \phi)\right| \leq \delta_{k i}
$$

That is, $x(t, \phi) \in \Psi$ for $t>0$.
We claim that $x(t, \phi)$ remains in $\Psi^{\eta}$ for any $t>0$. If it is not true, there exist $k \in I_{M}$ and $1 \leq i \leq l_{k}$ that $\left(x_{k i}(t), x_{(k+1) i}(t)\right)$ firstly (or one of the first) escapes from $\Psi_{k i}^{\eta_{k i}}$.

Case $1\left(w_{k i(k+1) i}, w_{(k+1) i k i}>0\right)$. If $\eta_{k i}=1$, then exists $t_{0}>0$, such that $\left(x_{k i}\left(t_{0}\right), x_{(k+1) i}\left(t_{0}\right)\right)$ is on the edges of $\Psi_{k i}^{\eta_{k i}}$, and for any $t<t_{0}, x_{k i}^{1-}<x_{k i}(t)<x_{k i}^{1+}, x_{(k+1) i}^{1-}<x_{(k+1) i}(t)<x_{(k+1) i}^{1+}$. If $x_{k i}\left(t_{0}\right)=x_{k i}^{1+}, x_{(k+1) i}\left(t_{0}\right) \in\left[x_{(k+1) i}^{1-}, x_{(k+1) i}^{1+}\right]$, then

$$
\begin{aligned}
\frac{\left.d x_{k i}(t)\right|_{t=t_{0}}=}{d t} & -a_{k i} x_{k i}\left(t_{0}\right)+\sum_{p=1, p \neq k}^{m} \sum_{j=1}^{n_{p}} w_{p j k i} f_{p j} \\
& \times\left(x_{p j}\left(t_{0}-\tau_{p j k i}\right)\right)+I_{k i} \\
\leq & -a_{k i} x_{k i}^{1+}+w_{(k+1) i k i} f_{(k+1) i}\left(x_{(k+1) i}^{1+}\right) \\
& -\left|w_{(k+1) i k i}\right| M_{(k+1) i} \\
& +\sum_{p=1, p \neq k}^{m} \sum_{j=1}^{n_{p}}\left|w_{p j k i}\right| M_{p j}+\left|I_{k i}\right| \\
= & -a_{k i}\left\{x_{k i}^{1+}-\frac{1}{a_{k i}}\left[w_{(k+1) i k i} f_{(k+1) i}\left(x_{(k+1) i}^{1+}\right)\right.\right. \\
= & -a_{k i}\left[x_{k i}^{1+}-G_{k i}^{+}\left(x_{(k+1) i}^{1+}\right)\right]=0 .
\end{aligned}
$$

Therefore, $\left(x_{k i}(t), x_{(k+1) i}(t)\right)$ cannot escape from $\Psi_{k i}^{\eta_{k i}}$ through the edge of $x_{k i}(t)=x_{k i}^{1+}, x_{(k+1) i}(t) \in\left[x_{(k+1) i}^{1-}, x_{(k+1) i}^{1+}\right]$. With similar proof, we can obtain that $\left(x_{k i}(t), x_{(k+1) i}(t)\right)$ cannot escape from $\Psi_{k i}^{\eta_{k i}}$ through the other three edges. Hence $\left(x_{k i}(t), x_{(k+1) i}(t)\right)$ cannot escape from $\Psi_{k i}^{1}$. We can also prove that $\left(x_{k i}(t), x_{(k+1) i}(t)\right)$ cannot escape from $\Psi_{k i}^{3}$.

Case $2\left(w_{k i(k+1) i}, w_{(k+1) i k i}<0\right)$. The proof is similar to that of Case 1.

This completes the proof.

We give the criterions concerning the stability for the multiple equilibria of system (1).

(H3) There exist constants $L_{k i}>0 \quad(1 \leq k \leq m$ and $1 \leq i \leq n_{k}$ ) such that

$$
\left|f_{k i}(x)-f_{k i}(y)\right| \leq L_{k i}|x-y|,
$$

for each $x, y$ in a subset $R_{k i} \subset \mathbb{R}$, where $R_{k i}$ is defined as follows:

$R_{k i}=$

$\begin{cases}{\left[x_{k i}^{1-}, x_{k i}^{1+}\right] \cup\left[x_{k i}^{3-}, x_{k i}^{3+}\right],} & \text { for } 1 \leq k \leq M, 1 \leq i \leq l_{k}, \\ {\left[\delta_{k i}^{-}, \delta_{k i}^{+}\right],} & \text {for } 1 \leq k \leq m, \quad l_{k}+1 \leq i \leq n_{k} .\end{cases}$

(H4) For any $1 \leq k \leq m, 1 \leq i \leq n_{k}$, it is satisfied that

$$
a_{k i}-\sum_{p=1, p \neq k j=1}^{m} \sum_{p j}^{n_{p}}\left|w_{p j k i}\right| L_{k i}>0 \text {. }
$$


Theorem 3. There are $2^{l}$ exponential stability equilibria of the multidirectional associative memory neural network (1), if the conditions (H1)-(H4) hold.

Proof. According to Theorem 1, for any $\alpha$, the multidirectional associative memory neural network (1) has an equilibrium in $\Omega^{\alpha}$; let it be $\bar{x}$. According to Lemma 2 , for any $\eta$, the solution $x(t, \phi)$ of the multidirectional associative memory neural network (1) is in $\Psi^{\eta}$ under initial condition $\phi \in \Psi^{\eta}$. Let

$$
y(t)=\operatorname{col}\left\{y_{k i}(t)\right\}=x(t, \phi)-\bar{x}
$$

System (1) becomes

$$
\begin{aligned}
& \frac{d y_{k i}(t)}{d t}=- \\
&+a_{k i} y_{k i}(t) \\
&+\sum_{p=1, p \neq k}^{m} \sum_{j=1}^{n_{p}} w_{p j k i}\left\{f_{p j}\left[y_{p j}\left(t-\tau_{p j k i}\right)+\bar{x}_{p j}\right]\right. \\
&\left.-f_{p j}\left(\bar{x}_{p j}\right)\right\},
\end{aligned}
$$

for $1 \leq k \leq m, 1 \leq i \leq n_{k}$.

According to condition (H4), there exists $\mu>0$ such that

$$
a_{k i}-\mu-\sum_{p=1, p \neq k}^{m} \sum_{j=1}^{n_{p}}\left|w_{p j k i}\right| L_{k i} e^{\mu \tau_{p j k i}}>0 .
$$

Define that $u_{k i}(t)=e^{\mu t}\left|y_{k i}(t)\right|$, for $1 \leq k \leq m$ and $1 \leq i \leq n_{k}$. It is obvious that $u_{k i}(t)>0$. Denote

$$
Q=\max _{1 \leq k \leq m, 1 \leq i \leq n_{k}}\left\{\sup _{\theta \in(-\tau, 0]}\left|x_{k i}(\theta)-\bar{x}_{k i}\right|\right\} .
$$

Let $\delta>1$ be an arbitrary real number. For any $\theta \in(-\tau, 0]$, $1 \leq k \leq m, 1 \leq i \leq n_{k}$, it is easy to obtain that $u_{k i}(t)<Q \delta$. We shall prove that

$$
u_{k i}(t)<Q \delta
$$

for $1 \leq k \leq m, 1 \leq i \leq n_{k}$, and any $t>0$. Suppose this is not the case; then there exist $k=q, i=r$, and a time $t_{0}$ such that $u_{k i}(t) \leq Q \delta$ for $t \in\left(-\tau, t_{0}\right], 1 \leq k \leq m$ and $1 \leq i \leq$ $n_{k}, u_{q r}\left(t_{0}\right)=Q \delta$, and $D^{+} u_{q r}\left(t_{0}\right) \geq 0$. From (30), we derive that

$$
\begin{aligned}
D^{+}\left|y_{q r}\left(t_{0}\right)\right| \leq & -a_{q r}\left|y_{q r}\left(t_{0}\right)\right| \\
& +\sum_{p=1, p \neq q}^{m} \sum_{j=1}^{n_{p}}\left|w_{p j q r}\right| L_{p j}\left|y_{p j}\left(t_{0}-\tau_{p j q r}\right)\right| .
\end{aligned}
$$

Hence, from (31) and (34),

$$
\begin{aligned}
& D^{+}\left|u_{q r}\left(t_{0}\right)\right| \\
& \leq \mu e^{\mu t_{0}}\left|y_{q r}\left(t_{0}\right)\right|+e^{\mu t_{0}} \\
& \times\left[-a_{q r}\left|y_{q r}\left(t_{0}\right)\right|+\right. \\
& \left.\sum_{p=1, p \neq q}^{m} \sum_{j=1}^{n_{p}}\left|w_{p j q r}\right| L_{p j}\left|y_{p j}\left(t_{0}-\tau_{p j q r}\right)\right|\right] \\
& =-\left(a_{q r}-\mu\right) e^{\mu t_{0}}\left|y_{q r}\left(t_{0}\right)\right| \\
& +\sum_{p=1, p \neq q}^{m} \sum_{j=1}^{n_{p}}\left|w_{p j q r}\right| L_{p j} e^{\mu \tau_{p j q r}}\left|u_{p j}\left(t_{0}-\tau_{p j q r}\right)\right| \\
& \leq-\left(a_{q r}-\mu\right) u_{q r}\left(t_{0}\right) \\
& +\sum_{p=1, p \neq q}^{m} \sum_{j=1}^{n_{p}}\left|w_{p j q r}\right| L_{p j} e^{\mu \tau_{p j q r}}\left[\sup _{\theta \in\left[t_{0}-\tau, t_{0}\right]} u_{p j}(\theta)\right] \\
& \leq-\left(a_{q r}-\mu-\sum_{p=1, p \neq q}^{m} \sum_{j=1}^{n_{p}}\left|w_{p j q r}\right| L_{p j} e^{\mu \tau_{p j q r}}\right) Q \delta \\
& \leq 0 \text {, }
\end{aligned}
$$

which is contradicting $D^{+} u_{q r}\left(t_{0}\right) \geq 0$. Hence the inequality (33) holds. Since $\delta>1$ is arbitrary, by allowing $\delta \rightarrow 1+$, we have $u_{k i}(t) \leq Q$. Therefore, $x(t, \phi)$ is exponentially convergent to $\bar{x}$.

Remark 4. The dynamic system (1) studied in this paper is different from the system in [18]. First, the coefficients of system (1) are constants, which are $T$-periodic functions in [18]. Second, the delays of system (1) are constant delays while they are distributed delays in [18]. Above all, our conclusions in this paper are different from those in [18], and the proof methods are different. In [18], we obtained the existence of $2^{n_{0}[\mathrm{~m} / 2]}$ exponentially stable $T$-periodic solutions, where $n_{0}=\min \left\{n_{1}, n_{2}, \ldots, n_{m}\right\}$ if $m$ is an even number or $n_{0}=$ $\min \left\{n_{1}, n_{2}, \ldots, n_{m-1}\right\}$ if $m$ is an odd number. But we obtain the existence of $3^{l}$ equilibria by Theorem 1 and the exponential stability of $2^{l}$ equilibria of them by Theorem 3 . Because $l_{k}=\min \left\{n_{k}, n_{k+1}\right\}$, so $n_{0}=\min \left\{l_{k} \mid k \in I_{M}\right\}$. It follows that $l=\sum_{k \in I_{M}} l_{k} \geq n_{0}[m / 2]$. Therefore, the number of equilibria obtained in this paper is more than that of [18] if the conclusions of [18] are used to the constant coefficient system (1). 

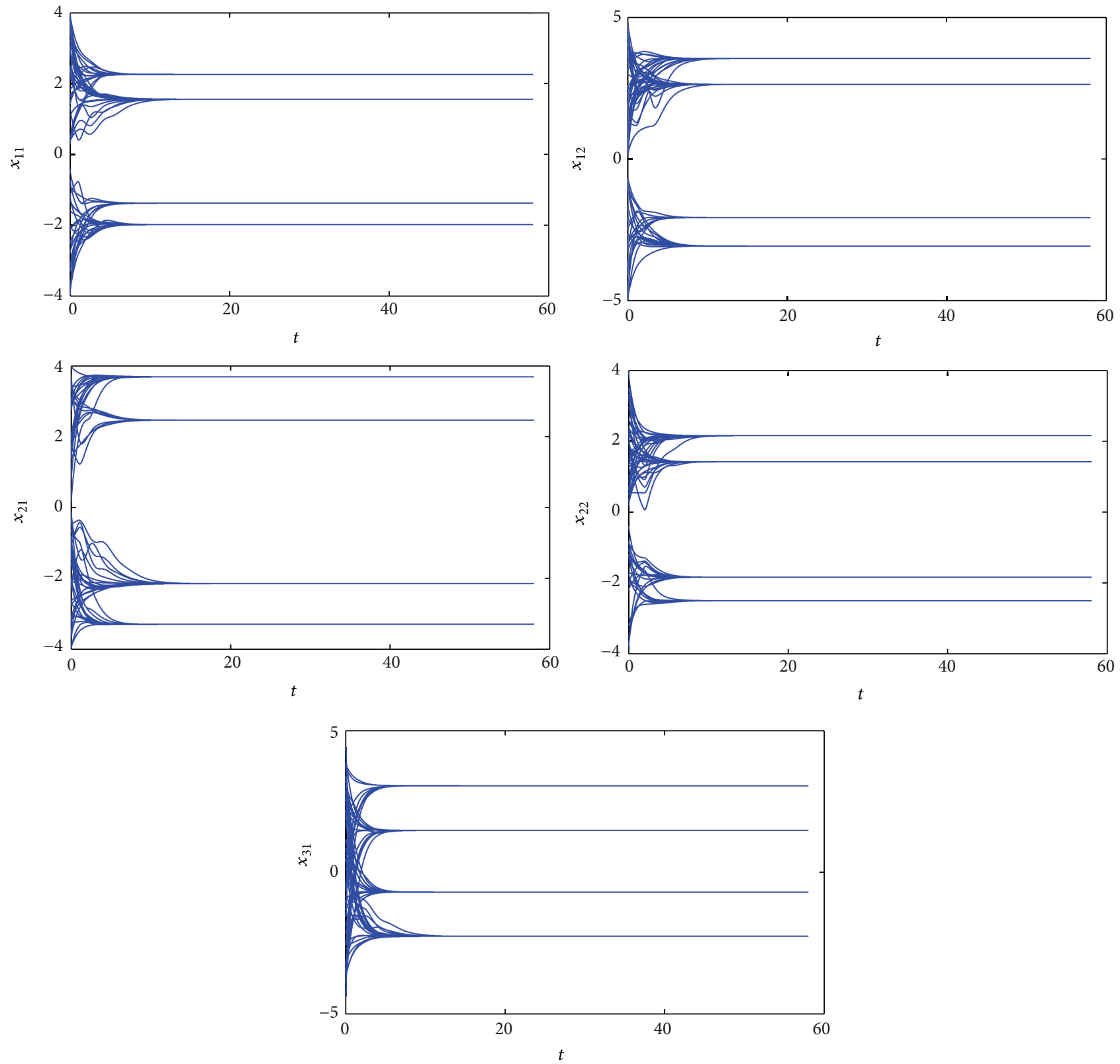

FIGURE 2: The exponential stability of 4 equilibria of the MAM neural network (36).

\section{Numerical Example}

In this section, a numerical example is given to illustrate the validity of results. Consider an MAM neural network with three fields as follows:

$$
\begin{aligned}
\frac{d x_{k i}}{d t}= & -a_{k i} x_{k i}(t)+\sum_{p=1, p \neq k}^{3} \sum_{j=1}^{n_{p}} w_{p j k i} f_{p j} \\
& \times\left(x_{p j}\left(t-\tau_{p j k i}\right)\right)+I_{k i},
\end{aligned}
$$

where $k=1,2,3, n_{1}=n_{2}=2, n_{3}=1$, the neuronal signal decay rates $a_{11}=a_{12}=a_{21}=a_{22}=a_{31}=1$, the external input $I_{11}=0.1, I_{12}=-0.4, I_{21}=0.2, I_{22}=-0.2, I_{31}=0.4$, the connection weights

$$
\begin{gathered}
\left(\begin{array}{ccccc}
0 & 0 & w_{2111} & w_{2211} & w_{3111} \\
0 & 0 & w_{2112} & w_{2212} & w_{3112} \\
w_{1121} & w_{1221} & 0 & 0 & w_{3121} \\
w_{1122} & w_{1222} & 0 & 0 & w_{3122} \\
w_{1131} & w_{1231} & w_{2131} & w_{2231} & 0
\end{array}\right) \\
=\left(\begin{array}{ccccc}
0 & 0 & -2 & 0.3 & 0.2 \\
0 & 0 & 0.4 & 3 & 0.1 \\
-3 & -0.5 & 0 & 0 & 0.1 \\
0.3 & 2 & 0 & 0 & -0.1 \\
1 & 2 & 1 & -1 & 0
\end{array}\right),
\end{gathered}
$$



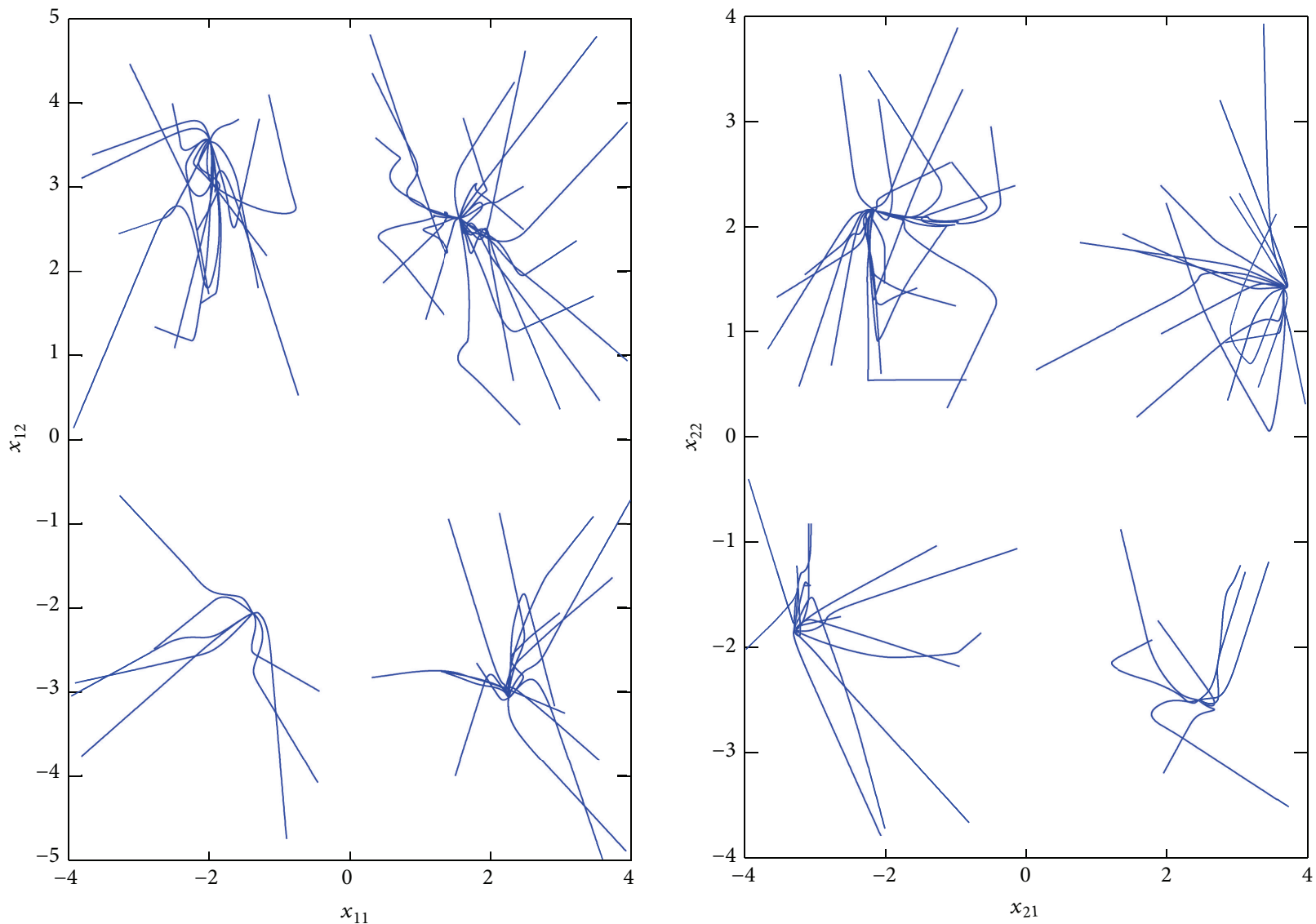

FIgURE 3: The phase of the MAM neural network (36).

and the delays

$$
\begin{gathered}
\left(\begin{array}{ccccc}
0 & 0 & \tau_{2111} & \tau_{2211} & \tau_{3111} \\
0 & 0 & \tau_{2112} & \tau_{2212} & \tau_{3112} \\
\tau_{1121} & \tau_{1221} & 0 & 0 & \tau_{3121} \\
\tau_{1122} & \tau_{1222} & 0 & 0 & \tau_{3122} \\
\tau_{1131} & \tau_{1231} & \tau_{2131} & \tau_{2231} & 0
\end{array}\right) \\
=\left(\begin{array}{ccccc}
0 & 0 & 1 & 2 & 1.2 \\
0 & 0 & 0.4 & 1 & 1.5 \\
1 & 0.4 & 0 & 0 & 0.8 \\
2 & 2 & 0 & 0 & 0.7 \\
1.2 & 1 & 1 & 2 & 0
\end{array}\right)
\end{gathered}
$$

The neuronal activation functions $f_{p j}(x)=\tanh (x)(1 \leq$ $\left.p \leq 3,1 \leq j \leq n_{p}\right)$ are continuous, increasing, and bounded functions, and there are $M_{p j}=1>0$ such that $\left|f_{p j}(x)\right| \leq$ $M_{p j}$. Hence condition (H1) is satisfied.

Obviously, $n=5, M=2, l_{1}=l_{2}=2$, and $l=2$. Through calculations, we have

$$
\begin{aligned}
& H_{21}^{ \pm}\left(x_{11}\right)=-3 f_{11}\left(x_{11}\right) \pm 0.8 \\
& G_{11}^{ \pm}\left(x_{21}\right)=-2 f_{21}\left(x_{21}\right) \pm 0.6 \\
& H_{22}^{ \pm}\left(x_{12}\right)=2 f_{21}\left(x_{12}\right) \pm 0.6 \\
& G_{12}^{ \pm}\left(x_{22}\right)=3 f_{22}\left(x_{22}\right) \pm 0.9
\end{aligned}
$$

There exist four points $\left(u_{11}^{1}, u_{21}^{1}\right)=(-0.8,1),\left(u_{11}^{2}, u_{21}^{2}\right)=$ $(0.9,-1.2),\left(u_{12}^{1}, u_{22}^{1}\right)=(-1,-0.9)$, and $\left(u_{12}^{2}, u_{22}^{2}\right)=(1.1,0.9)$, where $u_{11}^{1}<u_{11}^{2}, u_{12}^{1}<u_{12}^{2}$, such that

$$
\begin{aligned}
& H_{21}^{-}\left(u_{11}^{1}\right)=1.1921>1, \quad G_{11}^{+}\left(u_{21}^{1}\right)=-0.9232<-0.8, \\
& H_{21}^{+}\left(u_{11}^{2}\right)=-1.3489<-1.2, \quad G_{11}^{-}\left(u_{21}^{2}\right)=0.9673>0.9, \\
& H_{22}^{+}\left(u_{12}^{1}\right)=-0.9232<-0.9, \quad G_{12}^{+}\left(u_{22}^{1}\right)=-1.2489<-1, \\
& H_{22}^{-}\left(u_{12}^{2}\right)=1.0010>0.9, \quad G_{12}^{-}\left(u_{22}^{2}\right)=1.2489>1.1 .
\end{aligned}
$$

Hence condition (H2) holds. The curves $x_{21}=H_{21}^{+}\left(x_{11}\right)$, $x_{11}=G_{11}^{-}\left(x_{21}\right)$ have three crossing points $\left(x_{11}^{1-}, x_{21}^{1+}\right)=$ $(-2.5979,3.7669), \quad\left(x_{11}^{2+}, x_{21}^{2-}\right)=(0.5198,0.6327)$, and $\left(x_{11}^{3-}, x_{21}^{3+}\right)=(1.2886,-1.7763)$, and the curves $x_{21}=H_{21}^{+}\left(x_{11}\right), x_{11}=G_{11}^{-}\left(x_{21}\right)$ have three crossing points $\left(x_{11}^{1+}, x_{21}^{1-}\right)=(-1.2886,1.7763),\left(x_{11}^{2-}, x_{21}^{2+}\right)=(-0.5198$, $0.6327)$, and $\left(x_{11}^{3+}, x_{21}^{3-}\right)=(2.5979,-3.7669)$. The curves $x_{22}=H_{22}^{+}\left(x_{12}\right), x_{12}=G_{12}^{+}\left(x_{22}\right)$ have three crossing points $\left(x_{12}^{1+}, x_{22}^{1+}\right)=(-1.6517,-1.2582),\left(x_{12}^{2-}, x_{22}^{2-}\right)=$ $(-0.6830,-0.5869)$, and $\left(x_{12}^{3+}, x_{22}^{3+}\right)=(3.8670,2.5982)$, and the curves $x_{22}=H_{22}^{-}\left(x_{12}\right), x_{12}=G_{12}^{-}\left(x_{22}\right)$ have three crossing points $\left(x_{12}^{1-}, x_{22}^{1-}\right)=(-3.8670,-2.5982),\left(x_{12}^{2+}, x_{22}^{2+}\right)=$ 
$(0.6830,0.5869)$, and $\left(x_{12}^{3-}, x_{22}^{3-}\right)=(1.6517,1.2582)$. From (27), we obtain the subsets

$$
\begin{aligned}
& R_{11}=[-2.2579,-1.2886] \cup[1.2886,2.2579], \\
& R_{21}=[-3.7669,-1.7763] \cup[1.7763,3.7669], \\
& R_{12}=[-3.8670,-1.6517] \cup[1.6571,3.8670], \\
& R_{22}=[-2.5982,-1.2582] \cup[1.2582,2.5982], \\
& R_{31}=[-5.4,5.4] .
\end{aligned}
$$

Because the activation functions $f_{p j}(x)=\tanh (x)$ are differentiable, we can let $L_{p j}=\max \left\{f_{p j}^{\prime}(x) \mid x \in R_{p j}\right\}>0$; then $L_{11}=0.2625, L_{12}=0.1083, L_{21}=0.1368, L_{22}=0.2765$, and $L_{31}=1$. By Lagrange's mean value theorem, condition (H3) holds. Through calculations we have

$$
\begin{aligned}
& 1-2 L_{21}-0.3 L_{22}+0.2 L_{31}=0.4434>0, \\
& 1-0.4 L_{21}-3 L_{22}+0.1 L_{31}=0.0158>0, \\
& 1-3 L_{11}-0.5 L_{12}+0.1 L_{31}=0.0583>0, \\
& 1-0.3 L_{11}+2 L_{12}+0.1 L_{31}=0.6047>0, \\
& 1-L_{11}-2 L_{12}-L_{21}-L_{22}=0.1076>0 .
\end{aligned}
$$

Hence condition (H4) holds. Then by Theorems 1 and 3, the MAM neural network (36) has $3^{l}=9$ equilibria, and $2^{l}=4$ of these equilibria are exponentially stable.

The dynamics of the MAM neural network system (36) are illustrated in Figures 2 and 3. Evolutions of sixty initial conditions of the MAM neural network system (36) have been tracked in Figure 2, which clearly displays that there exist four stable equilibria of the dynamical system, as confirmed by our theorems. Figure 3 shows the phases of the evolutions from time 5 to time 60 with sixty initial conditions, which shows that each evolution has converged to one of the four stable equilibria at time 60 .

\section{Conclusions}

In this paper, the multistability has been studied for MAM neural networks. Sufficient conditions are obtained which ensure the existence of $3^{l}$ equilibria. It is proved that $2^{l}$ of the equilibria are exponentially stable. In [18], we have discussed the existence and the exponential stability of multiple periodic solutions for an MAM neural network. Furthermore, the coexistence of multiple stable equilibria and periodic solutions of an MAM neural network is an interesting topic. It will be investigated in the near future.

\section{References}

[1] B. Kosko, "Adaptove bidirectional associate memories," Applied Optics, vol. 26, no. 23, pp. 4947-4960, 1987.

[2] M. Hagiwara, "Multidirectional associative memory," in Proceedings of the International Joint Conference on Neural Networks, vol. 1, pp. 3-6, Washington, DC, USA, 1990.
[3] M. Hattori, M. Hagiwara, and M. Nakagawa, "Improved multidirectional associative memories for training sets including common terms," in Proceedings of the International Joint Conference on Neural Networks, vol. 2, pp. 172-177, Baltimore, Md, USA, 1992.

[4] M. Hattori and M. Hagiwara, "Multimodule associative memory for many-to-many associations," Neurocomputing, vol. 19, no. 1-3, pp. 99-119, 1998.

[5] M. Hattori and M. Hagiwara, "Associative memory for intelligent control," Mathematics and Computers in Simulation, vol. 51, no. 3-4, pp. 349-374, 2000.

[6] J. Huang and M. Hagiwara, "A combined multi-winner multidirectional associative memory," Neurocomputing, vol. 48, no. 1-4, pp. 369-389, 2002.

[7] D. Reich, K. Thangaraj, N. Patterson, A. L. Price, and L. Singh, "Reconstructing Indian population history," Nature, vol. 461, no. 7263, pp. 489-494, 2009.

[8] P. van den Driessche and X. Zou, "Global attractivity in delayed Hopfield neural network models," SIAM Journal on Applied Mathematics, vol. 58, no. 6, pp. 1878-1890, 1998.

[9] H. Zhao, "Global asymptotic stability of Hopfield neural network involving distributed delays," Neural Networks, vol. 17, no. 1, pp. 47-53, 2004.

[10] Y. Song, M. Han, and J. Wei, "Stability and Hopf bifurcation analysis on a simplified BAM neural network with delays," Physica D, vol. 200, no. 3-4, pp. 185-204, 2005.

[11] A. Chen, J. Cao, and L. Huang, "Exponential stability of BAM neural networks with transmission delays," Neurocomputing, vol. 57, no. 1-4, pp. 435-454, 2004.

[12] Q. Song, Z. Zhao, and Y. Li, "Global exponential stability of BAM neural networks with distributed delays and reactiondiffusion terms," Physics Letters A, vol. 335, no. 2-3, pp. 213-225, 2005.

[13] S. Chen and H. Gao, "Multivalued exponential multidirectional associative memory," Chinese Journal Software, vol. 9, pp. 397400, 1998.

[14] L. Chen, S.-C. Chen, and D.-Q. Zhang, "Small world structure inspired many to many kernel associative memory models and their application," Journal of Software, vol. 17, no. 2, pp. 223-231, 2006.

[15] T. Zhou, M. Wang, H. Fang, and X. Li, "Global exponential stability of MAM neural network with time delays," in Proceedings of the 5th International Conference on Bio-Inspired Computing: Theories and Applications (BIC-TA '10), pp. 6-10, Changsha, China, September 2010.

[16] M. Wang, T. Zhou, and H. Fang, "Global exponential stability of MAM neural network with time-varying delays," in Proceedings of the International Conference on Computational Intelligence and Software Engineering (CiSE '10), pp. 1-4, Wuhan, China, December 2010.

[17] M. Wang, T. Zhou, and X. Zhang, "Global exponential stability of discretetime multidirectional associative memory neural network with variable delays," ISRN Discrete Mathematics, vol. 2012, Article ID 831715, 10 pages, 2012.

[18] T. Zhou, M. Wang, and M. Long, "Existence and exponential stability of multiple periodic solutions for a multidirectional associative memory neural network," Neural Processing Letters, vol. 34, no. 2, pp. 187-202, 2012.

[19] L. Wang and T. Chen, "Coexistence and local stability of multiple equilibria in neural networks with piecewise linear nondecreasing activation functions," Neural Networks, vol. 23, no. 2, pp. 189-200, 2010. 
[20] L. Wang, W. Lu, and T. Chen, "Multistability of neural networks with Mexican-hat-type activation functions," IEEE Transactions Neural Networks Learning Systems, vol. 23, no. 11, pp. 1816-1826, 2012.

[21] T. Chen and L. Wang, "Complete stability of cellular neural networks with unbounded time-varying delays," Neural Networks, vol. 36, pp. 11-17, 2012.

[22] G. Huang and J. Cao, "Multistability in bidirectional associative memory neural networks," Physics Letters A, vol. 372, no. 16, pp. 2842-2854, 2008.

[23] Y. Huang, H. Zhang, and Z. Wang, "Dynamical stability analysis of multiple equilibrium points in time-varying delayed recurrent neural networks with discontinuous activation functions," Neurocomputing, vol. 91, pp. 21-28, 2012.

[24] S. A. Campbell, I. Ncube, and J. Wu, "Multistability and stable asynchronous periodic oscillations in a multiple-delayed neural system," Physica D, vol. 214, no. 2, pp. 101-119, 2006.

[25] C.-Y. Cheng, K.-H. Lin, and C.-W. Shih, "Multistability and convergence in delayed neural networks," Physica D, vol. 225, no. 1, pp. 61-74, 2007.

[26] G. Huang and J. Cao, "Multistability of neural networks with discontinuous activation function," Communications in Nonlinear Science and Numerical Simulation, vol. 13, no. 10, pp. 22792289, 2008.

[27] J. Cao, G. Feng, and Y. Wang, "Multistability and multiperiodicity of delayed Cohen-Grossberg neural networks with a general class of activation functions," Physica D, vol. 237, no. 13, pp. 1734-1749, 2008.

[28] L. Chandrasekaran, V. Matveev, and A. Bose, "Multistability of clustered states in a globally inhibitory network," Physica D, vol. 238, no. 3, pp. 253-263, 2009.

[29] X. Nie and J. Cao, "Multistability of competitive neural networks with time-varying and distributed delays," Nonlinear Analysis: Real World Applications, vol. 10, no. 2, pp. 928-942, 2009.

[30] X. Nie and Z. Huang, "Multistability and multiperiodicity of high-order competitive neural networks with a general class of activation functions," Neurocomputing, vol. 82, pp. 1-13, 2012.

[31] Z. Zeng and W. Zheng, "Multistability of neural networks with time-varying delays and concave-convex characteristics," IEEE Transactions on Neural Networks and Learning System, vol. 23, no. 2, pp. 293-305, 2012.

[32] Y. Du, Y. Li, and R. Xu, "Multistability and multiperiodicity for a general class of delayed Cohen-Grossberg neural networks with discontinuous activation functions," Discrete Dynamics in Nature and Society, Article ID 917835, 11 pages, 2013. 


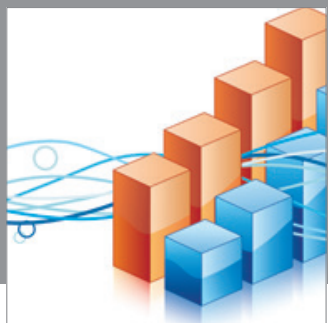

Advances in

Operations Research

mansans

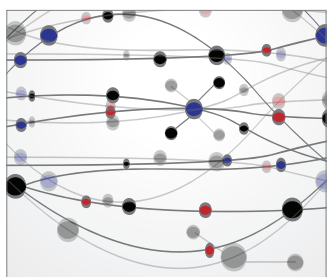

The Scientific World Journal
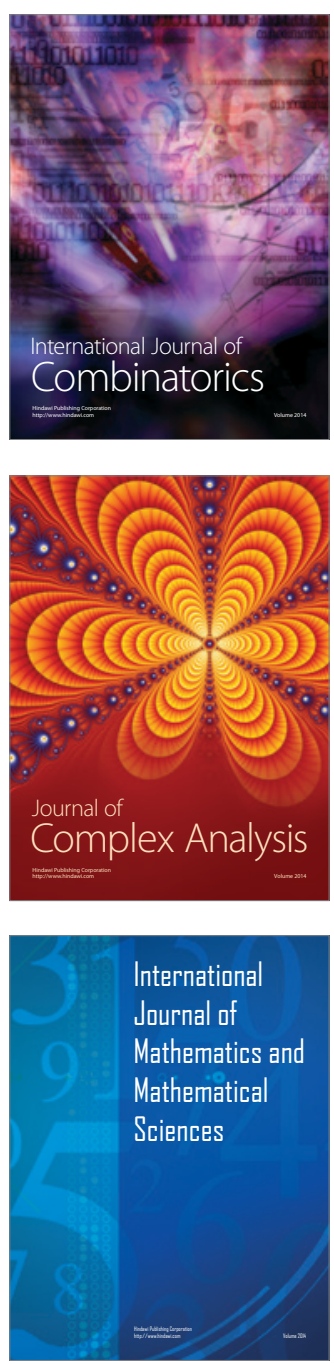
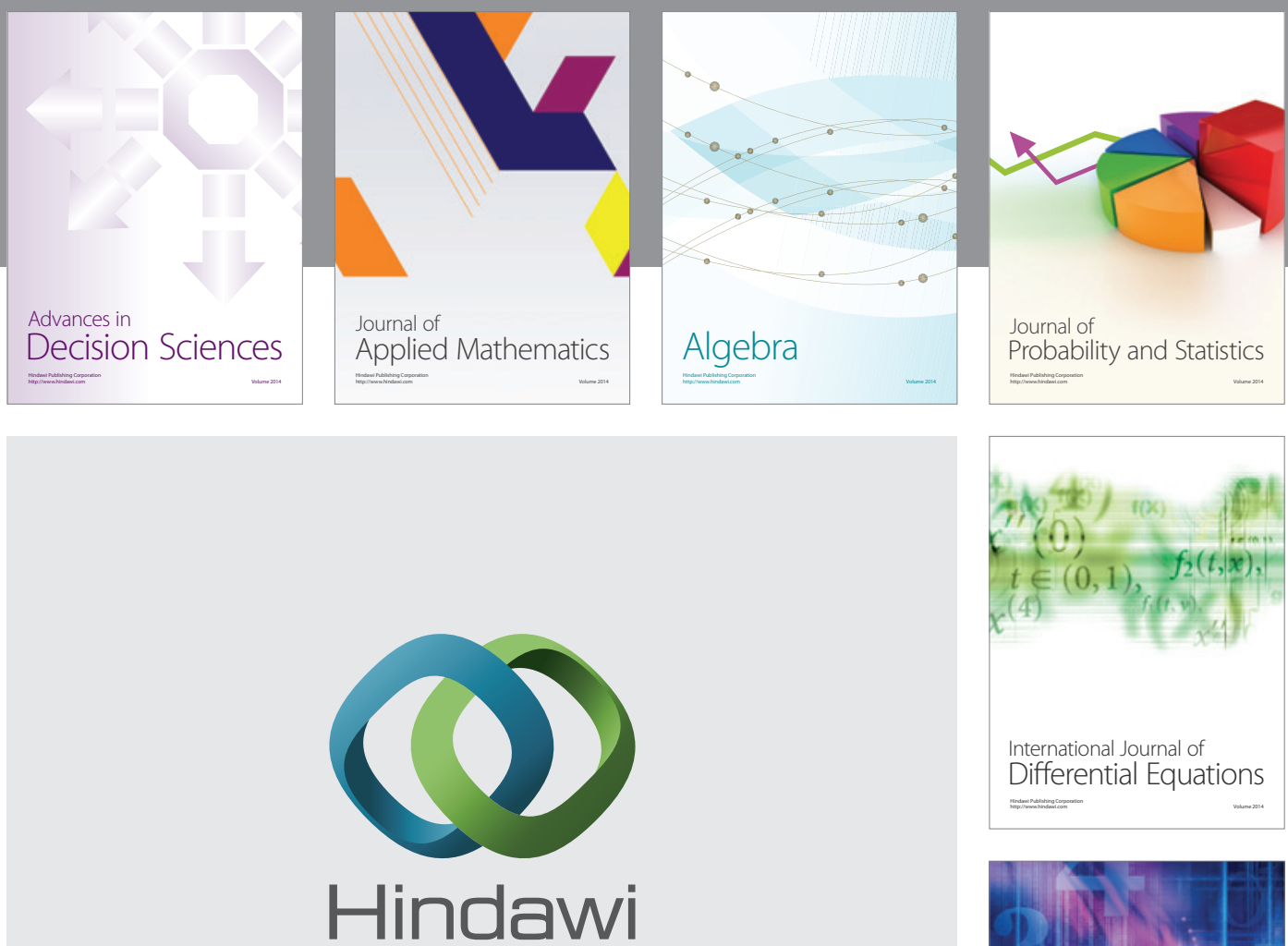

Submit your manuscripts at http://www.hindawi.com
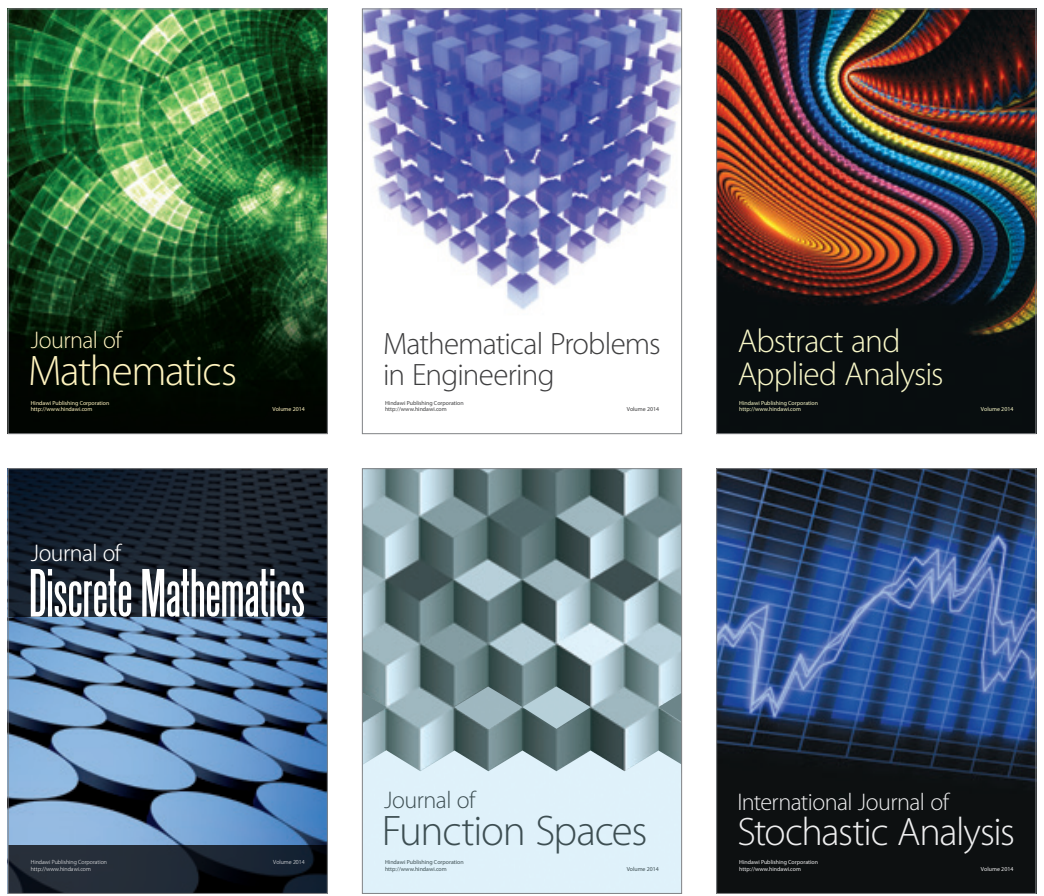

Journal of

Function Spaces

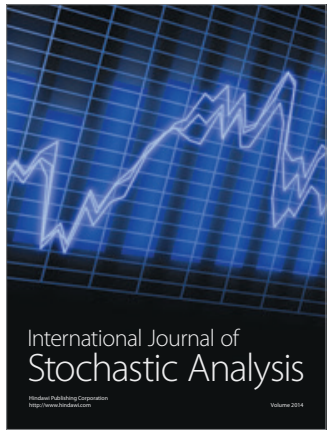

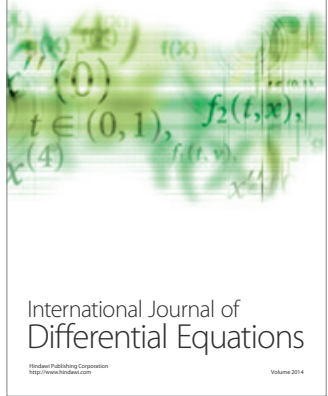
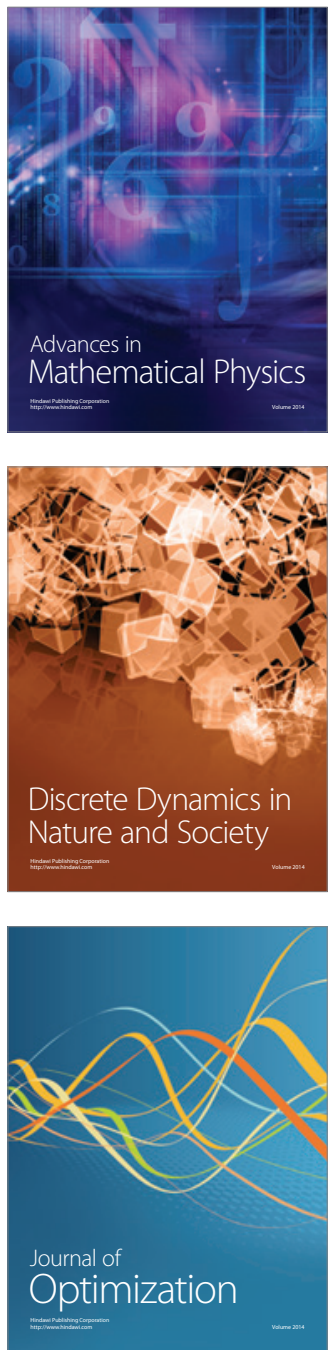